\title{
Role of Preload in Adhesion of Rough Surfaces
}

\author{
L. Dorogin, ${ }^{1,2,3}$ A. Tiwari, ${ }^{4,2}$ C. Rotella, ${ }^{5}$ P. Mangiagalli, ${ }^{5}$ and B. N. J. Persson ${ }^{2, *}$ \\ ${ }^{1}$ Leibniz Institute for Polymer Research Dresden, P.O. Box 120 411, D-01005 Dresden, Germany \\ ${ }^{2}$ Peter Grünberg Institute, Forschungszentrum Jülich, 52425 Jülich, Germany \\ ${ }^{3}$ ITMO University, Kronverskiy pr. 49, 197101 Saint Petersburg, Russia \\ ${ }^{4}$ Department of Mechanical and Industrial Engineering, Norwegian University of Science and Technology, \\ Richard Birkelandsvei 2B, N-7491 Trondheim, Norway \\ ${ }^{5}$ Sanofi, 13, quai Jules Guesde-BP 14-94403 Vitry sur Seine Cedex, France
}

(Received 8 March 2017; published 9 June 2017)

\begin{abstract}
Adhesion between glass and bromobutyl and polydimethylsiloxane elastomers is investigated. We show that viscoelastic energy dissipation close to the opening (or closing) crack tip, and surface roughness, strongly affect the work of adhesion. We observe strong adhesion hysteresis and we show, in contrast to the Johnson-Kendall-Roberts theory prediction for elastic solids, that this results in a pull-off force, and effective work of adhesion to be dependent on the maximum loading force.
\end{abstract}

DOI: 10.1103/PhysRevLett.118.238001

Introduction.-Contact mechanics and adhesion are the central topics in tribology [1-3] with applications to tires, seals, human joints, pressure sensitive adhesives, granular matter, wiper blades and syringes, to name just a few. Contact mechanics for elastic solids with randomly rough surfaces in the absence of adhesion is now rather well explored [4-10]. However, with adhesion the problem becomes much more complex and not fully understood [11-19], in particular, for real materials like rubber with viscoelastic and nonlinear properties [20-25].

The simplest and most well-defined contact mechanics problem is the contact between a ball (radius $R$ ) and a flat surface. For an elastically soft contact the Johnson-KendallRoberts (JKR) [26,27] theory is valid. This theory can predict the pull-off force $F=(3 \pi / 2) w R$, where $w$ is the work of adhesion. In the adiabatic limit (i.e., infinitesimally low pull-off velocity) $w$ is equal to the change in the interfacial free energy $\Delta \gamma=\gamma_{1}+\gamma_{2}-\gamma_{12}$ (where $\gamma_{1}, \gamma_{2}$, and $\gamma_{12}$ is the solid-vapor interfacial energies of solid 1 and 2 , and the interfacial energy of the contact between solid 1 and 2, respectively). However, for pull off at a finite speed the work of adhesion is often much higher than $\Delta \gamma$ due to the nonadiabatic effects.

The JKR theory assumes perfectly smooth surfaces. The contact between elastic solids with random roughness on many length scales is a much more complex topic, involving asperities with radius of curvature extending over many decades in length scale. However, if there is a separation in length scales, where the diameter of the nominal ball-substrate contact region is larger than the most long-wavelength surface roughness component, then the JKR theory is still valid, but with $\Delta \gamma$ replaced by an effective interfacial binding energy that depends on the surface roughness.

The JKR theory is also valid for viscoelastic solids such as rubber, assuming the loading and pull off occur so slowly that the material can be considered as purely elastic far from the (circular) contact-non-contact boundary line (crack tip). However, in general, the work of adhesion during pull off is strongly influenced by two competing effects: nonadiabatic effects, in particular the viscoelastic energy dissipation in the vicinity of the opening crack tip $[23,28]$, which may strongly increase the work of adhesion, and the surface roughness, which usually reduces the work of adhesion [29]. The nonadiabatic effects also often result in an adhesion force that is much smaller during approach than during pull off, an effect referred to as adhesion hysteresis.

The JKR theory, when applied to systems with smooth surfaces, predicts that the pull-off force is independent of the magnitude of the maximal normal force (the load). This results holds also for viscoelastic solids if complete contact occurs in the nominal contact region. We will show in this Letter, however, that if only partial contact occur at the interface (which is almost always the case in practical applications due to surface roughness), the pull-off force will increase with increasing loading force. This fact opens up the possibility to gain information about the area of real contact from the load dependency of the work of adhesion deduced from the JKR theory.

Short review of the JKR theory.-The contact region between a rigid spherical probe (radius $R$ ) and a flat elastomer surface is circular with the radius $r$. In the JKR theory, the interaction between the solids is described by the work of adhesion $w$, which is the energy per unit surface area necessary to separate two flat surfaces from their equilibrium contact position to infinite separation. According to the JKR theory the relation between the interaction force $F$, and the radius $r$ on the stable branch of the interaction curve is $[26,27]$

$$
r^{3}=\frac{3 R F_{\mathrm{c}}}{4 E^{*}}\left[\frac{F}{F_{c}}+2+2\left(\frac{F}{F_{c}}+1\right)^{1 / 2}\right]
$$


where $E^{*}=E /\left(1-\nu^{2}\right), E$ and $\nu$ are the elastomer Young's modulus and Poisson ratio, respectively, and

$$
F_{c}=\frac{3 \pi}{2} w R,
$$

is the pull-off force. Thus, for an elastic solid, if the ball is pulled by a soft spring and neglecting the inertia effects, at $F=-F_{c}$ the pull-off force abruptly drops to zero.

The separation line $r=r(t)$ can be considered as a crack tip [30,31]. The work of adhesion $w$ in general depends on the velocity $v_{r}=\dot{r}$ of the opening (during pull-off) or closing (during contact formation) crack tip. At finite crack tip velocity, for an opening crack $w$ can be strongly enhanced, and for a closing crack strongly reduced, compared to the adiabatic (infinitely low crack tip velocity) value $w_{0}$.

The JKR theory reduces to Hertz contact mechanics theory when the work of adhesion vanishes. In this limit the maximum contact pressure $p_{0}$ as well as the average one depend on the loading force $F$,

$$
p_{0}=\left(\frac{6 F E^{* 2}}{\pi^{3} R^{2}}\right)^{1 / 3}
$$

In the case of surface roughness only partial contact occurs within the nominal Hertz contact area. In particular, if the relative contact area $A / A_{0}<0.3$, the true contact area $A$ will depend linearly on the (nominal) contact pressure $p$ (see Refs. [5,6,8]):

$$
\frac{A}{A_{0}}=\frac{\kappa p_{0}}{E^{*} h^{\prime}},
$$

where $h^{\prime}$ is the root-mean-square surface slope, and where $\kappa \approx 2.0$. Combing Eqs. (3) and (4) shows that if $A / A_{0}<0.3$ the relative contact area is proportional to $F^{1 / 3}$, a result we will make use of later.

The JKR theory is based on the assumption of the contact between a sphere and an elastic half-space. For the system studied below (1) predict that the radius of the contact region at the point of the snap-off instability is of order $1 \mathrm{~mm}$ for the highest preload, while the thickness of the rubber sheet is about $3 \mathrm{~mm}$. Thus, we expect the half-space approximation to hold reasonably accurately.

Experiment.-We study the adhesion interaction between a spherical soda-lime glass ball (diameter $2 R=4 \mathrm{~cm}$ ) and an elastomer. We bring the ball into contact with the substrate using a drive that can be represented by a spring. The contact region is not observed directly but only the time dependency of the interaction force $F(t)$, from which we can calculate the crack tip velocity $v_{r}=\dot{r}(t)$ using Eq. (1).

The rubber substrate is positioned on a precise analytical balance (analytical balance produced by Mettler Toledo, model MS104TS/00), which has a reproducibility of $0.1 \mathrm{mg}$ (or $\approx 1 \mu \mathrm{N}$ ) (see Fig. 1). After zeroing the scale of the instrument we can measure the force $F(t)$ on the substrate as a function of time, which is directly transferred to a computer at the rate of 1 sample per second [32].

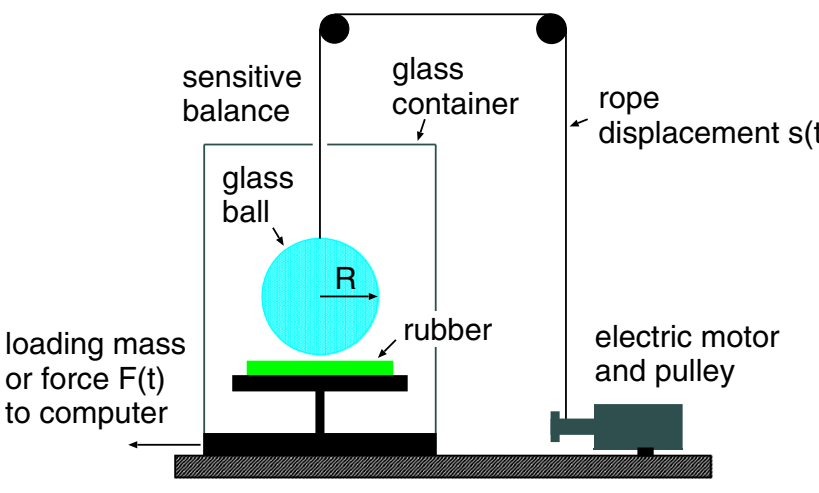

FIG. 1. The experimental set up for measuring adhesion.

To move the glass ball up and down we use an electric motor coiling up a nylon cord, which is attached to the glass ball. The drive velocity as a function of time can be specified electronically.

We consider the adhesion between soda-lime glass balls and carbon black filled bromobutyl rubber (supplied by West Pharmaceutical Services) and polydimethylsiloxane (PDMS). For the bromobutyl rubber we have studied the surface topography using an optical method and atomic force microscopy (AFM). The surface has a Gaussian height probability distribution with the root mean square (rms) roughness of about $h_{\text {rms }} \approx 2.9 \pm 0.1 \mu \mathrm{m}$, and with the highest point $\sim 13 \mu \mathrm{m}$ above the average plane. The surface roughness power spectrum is well approximated with that of a self-affine fractal surface with the Hurst exponent $H=$ 0.92 (or fractal dimension $D_{f}=2.08$ ). The power spectrum corresponds to a surface with the rms slope $h^{\prime}=0.55$.

The PDMS elastomer was produced from Sylgard 184. This is a two-component kit purchased from Dow Corning (Midland, MI) consisting of a base (vinyl-terminated polydimethylsiloxane) and a curing agent (methylhydrosiloxane-dimethylsiloxane copolymer) with a suitable catalyst. From these two components mixtures $1: 10$ (cross-linker:base) in weight were prepared. The mixture was degassed to remove the trapped air induced by stirring from the mixing process and then poured into the cast. The samples were cured in an oven at $80^{\circ} \mathrm{C}$ for $14 \mathrm{~h}$. The PDMS upper surface was very smooth due to gravity, and for the present study we consider it as perfectly smooth.

The linear and nonlinear viscoelastic properties for the bromobutyl and PDMS elastomer were obtained using a Q800 Dynamic Mechanical Analysis (DMA) instrument produced by TA Instruments, following the procedure described elsewhere [33].

Interfacial crack propagation.-The contact line between a spherical probe and an elastomer substrate can be considered as a crack tip and the work of adhesion $w$ equals the crack propagation energy per unit surface area. It is known that the crack propagation energy depends on the crack tip velocity $v$ and on the temperature $T$, i.e., $w=w(v, T)$. In addition, it differs for a closing crack and an opening crack. 


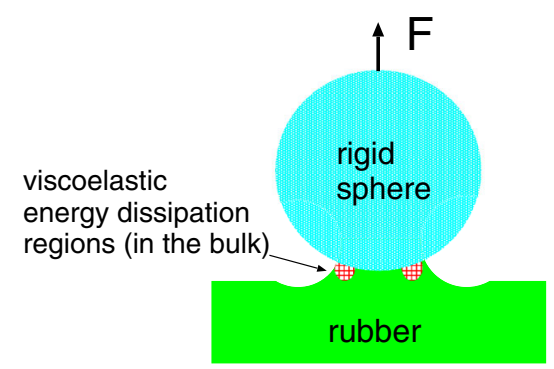

FIG. 2. A rigid ball pulled away from a viscoelastic solid. A part of the energy needed to remove the ball is derived from the viscoelastic energy dissipation inside the rubber close to the opening crack tip (red dashed region).

One contribution to the crack propagation energy (or work of adhesion) is derived from the viscoelastic energy dissipation in the vicinity of the crack tip (see Fig. 2). For an opening crack this will enhance $w$ with a factor usually denoted as $1+f\left(v_{r}, T\right)$. For a closing crack the corresponding reduction factor is approximately $[34] \approx 1 /\left[1+f\left(v_{r}, T\right)\right]$.

The crack propagation energy for an opening crack is often written as [28,31,35-38]:

$$
w(v, T)=w_{0}[1+f(v, T)] .
$$

Here, we are interested in interfacial (between the glass ball and the rubber substrate) crack propagation. In this case, as the crack velocity $v \rightarrow 0$ (when viscous effects in the rubber are negligible), the measured value of $w_{0}$ can be identified as the energy $w_{0}=\Delta \gamma=\gamma_{1}+\gamma_{2}-\gamma_{12}$ needed to break the interfacial rubber-substrate bonds, which are usually of the van der Waals type.

The factor $f(v, T)$ depends on the viscoelastic energy dissipation inside the elastomer close to the opening crack tip (red dashed region in Fig. 2). It can be calculated from the measured viscoelastic modulus as described in Refs. [23,28]. For the bromobutyl rubber at $T=20^{\circ}$ and at the crack tip velocity $v \approx 100 \mu \mathrm{m} / \mathrm{s}$ relevant for the study below we get the viscoelastic enhancement factor $[1+f(v, T)] \approx 60$. Thus, we expect strong adhesion hysteresis where the work of adhesion is enhanced by a factor of $\approx 60$ during pull off and reduced by a factor $1 /[1+$ $f(v, T)] \approx 1 / 60$ during approach. This is in good agreement with the measured data presented below where we do not observe any adhesion during approach while during pull off the adhesion is much stronger than expected assuming the adiabatic work of adhesion $w_{0}$.

Adhesion.-We first assume that the loading and unloading velocities are very small so that viscoelastic effects, and other nonadiabatic processes, are unimportant. In this limit, the adhesive contact is characterized by the adiabatic work of adhesion (for perfectly flat surfaces) $w_{0}=\Delta \gamma=\gamma_{1}+\gamma_{2}-\gamma_{12}$.

Let us estimate the adiabatic work of adhesion $w_{0}$ between the bromobutyl rubber and the glass surface. The surface energy (per unit surface area) for glass cleaned with acetone [which results in a surface still covered by water and some (strongly bounded) organic contamination] is typically $\gamma_{1} \approx 0.06-0.07 \mathrm{~J} / \mathrm{m}^{2}$. The surface energy for bromobutyl rubber is [39] $\gamma_{2} \approx 0.025-0.035 \mathrm{~J} / \mathrm{m}^{2}$. In a simple approach one assumes that the adiabatic work of adhesion is [3] $w_{0} \approx 2\left(\gamma_{1} \gamma_{2}\right)^{1 / 2}$, which in the present case gives $w_{0} \approx 0.08-0.1 \mathrm{~J} / \mathrm{m}^{2}$.

Using the work of adhesion $w_{0}=0.1 \mathrm{~J} / \mathrm{m}^{2}$ and the measured surface roughness power spectrum of the bromobutyl rubber, the theory presented in Ref. [29] shows that the macroscopic effective interfacial binding energy $\gamma_{\text {eff }}$ vanishes, and, hence, one does not expect any adhesion force on approach (contact formation) or pull off in the adiabatic limit. Thus, if there would be no adhesion hysteresis, the calculations show that the pull-off force would vanish for the glass ball in contact with the bromobutyl rubber.

Let us now include the adhesion hysteresis. We will show that the pull-off force can be finite when adhesion hysteresis prevails. Assume that at the end of the loading cycle the asperity contact regions appear as shown in Fig. 3(a). In the absence of adhesion, during unloading the asperity contact regions would disappear in a similar way as they were formed, and asperity contact regions even at the center of the macroscopic contact area will decrease in size even at the start of unloading, where the radius $r(t)$ of the macroscopic separation line (dashed lines in Fig. 3) is far from the center of the contact region. However, if the adhesion hysteresis is large enough so that the energy per unit area for the opening crack $w(v) \gg \Delta \gamma$, then the asperity contact regions will only

(a)

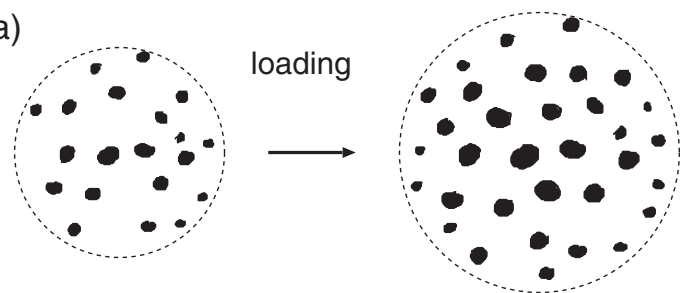

(b)

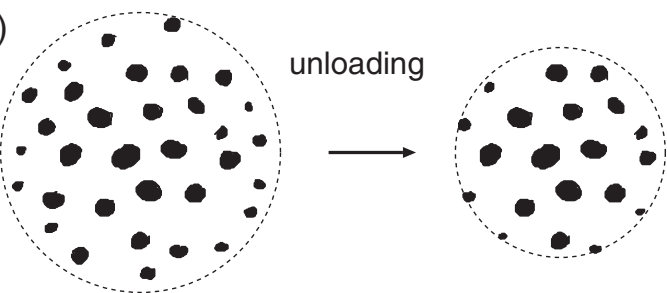

FIG. 3. (a) Schematic picture of the macroscopic contact area during loading, and (b) during unloading when strong adhesion hysteresis occurs so that $w(v) \gg \Delta \gamma$. The black regions indicate asperity contact regions. Because of the adhesive interaction, complete contact occurs within the black regions. During pull off in the case of strong adhesion hysteresis, the size of the asperity contact regions remains unchanged (in spite of the reduction in the contact pressure) except close to the macroscopic (apparent) opening crack tip (dashed circle), where the asperity contact regions are broken by the propagation of microscopic opening cracks at each asperity contact region. 
start to shrink when they are very close to the macroscopic opening crack (in the crack-tip process zone) [Fig. 3(b)]. In this case the pull-off force will be nonvanishing, and to a good approximation given by the JKR theory with $w_{\text {rough }} \approx w(v) A / A_{0}$, where $w(v)$ is the work of adhesion (for opening crack) obtained from the contact between smooth surfaces, and $A / A_{0}$ the relative area of real contact (at the maximum load at the radial distance from the center of the contact region where the detachment instability occurs). This is illustrated in Fig. 3(b), where the size of the asperity contact regions outside of the crack-tip process zone remain unchanged (in spite of the reduction in the contact pressure). Very close to the tip of the macroscopic (apparent) opening crack (dashed circle), the asperity contact regions are broken by the propagation of microscopic opening cracks at each asperity contact region.

We now present experimental results for how the pull-off force $F_{\text {pull-off }}$ depends on the applied load or maximum normal force $F_{m}$. Figure 4(a) shows the interaction force as
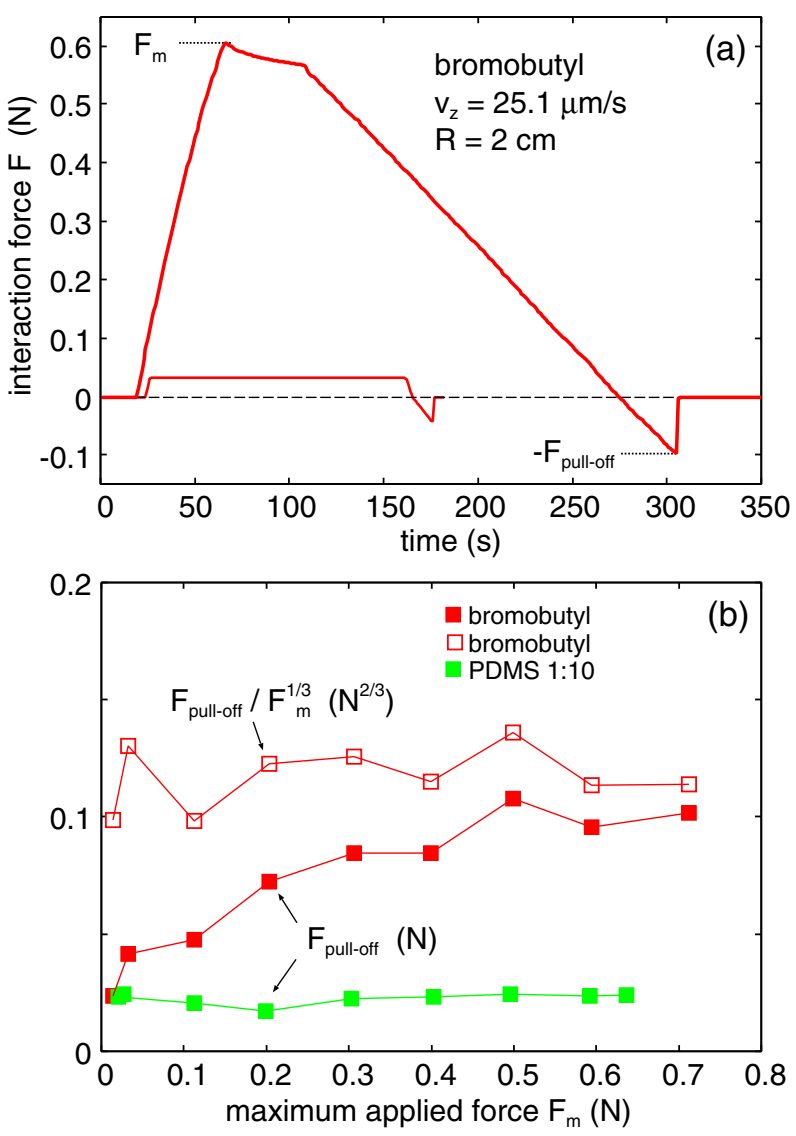

FIG. 4. (a) The interaction force between the bromobutyl rubber and the glass ball for two different loading situations (thin and thick solid lines), as a function of time. The glass ball approach and retraction velocity $v_{z}= \pm 25 \mu \mathrm{m} / \mathrm{s}$. (b) Filled squares: the pull-off force $F_{\text {pull-off }}$, as a function of the maximum loading force $F_{m}$, for bromobutyl rubber (read squares) and for PDMS (green squares). The open red squares is the pull-off force $F_{\text {pull-off }}$ divided by $F_{m}^{1 / 3}$ for bromobutyl rubber, as a function of $F_{m}$. For a glass ball with diameter $2 R=4 \mathrm{~cm}$. a function of time when a glass ball (diameter $2 R=4 \mathrm{~cm}$ ) approached and was retracted (speed $v_{z}= \pm 25 \mu \mathrm{m} / \mathrm{s}$ ) from a sheet of the bromobutyl rubber. We show results for two different loading situations, where the maximum loading forces are $F_{m}=0.59$ (thick line) and $0.033 \mathrm{~N}$ (thin line). The maximum loading force used in our study was $F_{m}=0.71 \mathrm{~N}$. Assuming the rubber elastic modulus $E_{\text {eff }}=2.5 \mathrm{MPa}$, as obtained from the DMA measurements, gives the Hertz maximum contact pressures [from Eq. (3)] $p_{0}=0.23 \mathrm{MPa}$ and the maximum relative contact area [from Eq. (4)]: $A / A_{0} \approx 0.16$. Thus, all our measured data are in the region where, in the absence of adhesion, the theory predicts that the contact area depends linearly on the contact pressure. Thus, since the Hertz contact pressure scales as $F_{m}^{1 / 3}$, we expect $A / A_{0}$, and hence also the pull-off force [40], to be proportional to $F_{m}^{1 / 3}$. Figure 4(b) shows that this is indeed the case for bromobutyl rubber.

The measured pull-off force $F_{\text {pull-off }}=0.1 \mathrm{~N}$ when the load $F_{m}=0.71 \mathrm{~N}$ [see Fig. 4(b)]. Using Eq. (2) this gives $w_{\text {rough }}=1.06 \mathrm{~J} / \mathrm{m}^{2}$ and from $w_{\text {rough }} \approx w(v) A / A_{0}$ we obtain $w(v)=6.6 \mathrm{~J} / \mathrm{m}^{2}$. Here we have used the relative contact area $A / A_{0}=0.16$ as calculated at the maximum preload and without adhesion, since adhesion is unimportant during contact formation because of the strong adhesion hysteresis (see above). Next, note that $w(v)=w_{0}[1+f(v, T)]$ and using the calculated viscoelastic enhancement factor $[1+$ $f(v, T)] \approx 60$ we get $w_{0} \approx 0.1 \mathrm{~J} / \mathrm{m}^{2}$. This agrees with the adiabatic work of adhesion estimated above. This shows that other nonadiabatic processes, which could contribute to the work of adhesion at finite crack tip speeds, are negligible compared to the viscoelastic contribution. In particular, it has been suggested that stretching of polymer chains before breaking the polymer-substrate bond could enhance the work of adhesion during pull-off $[19,41,42]$. Similarly, for surfaces with roughness, local elastic instabilities can enhance the work of adhesion during pull-off [43-47]. However, the analysis above shows that at least for the present system the main (nonadiabatic) enhancement of the work of adhesion result from the viscoelastic contribution.

In Ref. [48] it was found that for PDMS, in particular, for weakly cross-linked PDMS, the contribution to the work of adhesion from the bulk viscoelastic energy dissipation is not enough to explain the strong increase in the crack propagation energy for large enough pull-off speeds, and in these cases the processes considered in Refs. [19,41,42] may be involved in the crack-tip process zone, and contribute to the observed adhesion hysteresis. Clearly, in general, the processes occurring on a molecular scale at the adhesive interface will depend on many factors such as elastomer molecular chain segment mobility (at the interface), energetic barriers for bond formation, surface contamination, contact time, crack tip speed, and the temperature. However, the effect we study in this Letter does not depend on the exact origin of the adhesion hysteresis. 
For smooth surfaces, where the contact is complete, we do not expect any dependency of the pull-off force on the maximum loading force. To test this we performed experiments using a PDMS elastomer sheet with very smooth surface. Figure 4(b) shows results for the PDMS elastomer, where complete contact between the rubber and the glass will prevail within the nominal contact area. In this case, as expected, the pull-off force is independent of the maximum loading force.

In conclusion, we have shown that the work of adhesion of a contact with a rough surface can be enhanced by increasing the maximum preload force. This effect is deeply related to the adhesion hysteresis that occurs in the condition of the incomplete contact induced by the roughness. The observation of the load dependence can provide an insight in the contact mechanics of JKR-type experiments.

The research work was performed within a ReinhartKoselleck project funded by the Deutsche Forschungsgemeinschaft (DFG). We would like to thank the DFG for the project support under the reference German Research Foundation DFG-Grant: MU 1225/36-1. The research work was also supported by the DFG-Grant: PE 807/10-1. This work is supported in part by the Research Council of Norway (Project No. 234115 in the Petromaks2 programme) and by COST Action MP1303.

*www.MultiscaleConsulting.com

[1] B. N. J. Persson, Sliding Friction: Physical Principles and Applications (Springer, Heidelberg, 2000).

[2] E. Gnecco and E. Meyer, Elements of Friction Theory and Nanotribology (Cambridge University Press, Cambridge, England, 2015).

[3] J. N. Israelachvili, Intermolecular and Surface Forces, 3rd ed. (Academic, London, 2011).

[4] B. N. J. Persson, Surf. Sci. Rep. 61, 201 (2006).

[5] S. Hyun, L. Pei, J.-F. Molinari, and M. O. Robbins, Phys. Rev. E 70, 026117 (2004).

[6] C. Campana and M. H. Müser, Europhys. Lett. 77, 38005 (2007).

[7] W. B. Dapp, A. Lucke, B. N. J. Persson, and M. H. Müser, Phys. Rev. Lett. 108, 244301 (2012).

[8] B. N. J. Persson, J. Chem. Phys. 115, 3840 (2001).

[9] S. Akarapu, T. Sharp, and M. O. Robbins, Phys. Rev. Lett. 106, 204301 (2011).

[10] C. Campana, B. N. J. Persson, and M. H. Müser, J. Phys. Condens. Matter 23, 085001 (2011).

[11] L. Pastewka and M. O. Robbins, Proc. Natl. Acad. Sci. U.S.A. 111, 3298 (2014).

[12] B. N. J. Persson, I. M. Sivebaek, V. N. Samoilov, K. Zhao, A. I. Volokitin, and Z. Zhang, J. Phys. Condens. Matter 20, 395006 (2008).

[13] G. Carbone, M. Scaraggi, and U. Tartaglino, Eur. Phys. J. E 30, 65 (2009).

[14] A. Chateauminois and C. Fretigny, Eur. Phys. J. E 27, 221 (2008).
[15] B. Z. Newby, M. K. Chaudhury, and H. R. Brown, Science 269, 1407 (1995).

[16] D. L. Schmidt, R. F. Brady, K. Lam, D. C. Schmidt, and M. K. Chaudhury, Langmuir 20, 2830 (2004).

[17] M. K. Chaudhury, Mater. Sci. Eng. R 16, 97 (1996).

[18] P. Silberzan, S. Perutz, E. J. Kramer, and M. K. Chaudhury, Langmuir 10, 2466 (1994).

[19] A. Ghatak, K. Vorvolakos, H. She, D. L. Malotky, and M. K. Chaudhury, J. Phys. Chem. B 104, 4018 (2000).

[20] B. Lorenz, B. A. Krick, N. Mulakaluri, M. Smolyakova, S. Dieluweit, W. G. Sawyer, and B. N. J. Persson, J. Phys. Condens. Matter 25, 225004 (2013).

[21] B. A. Krick, J. R. Vail, B. N. J. Persson, and W. G. Sawyer, Tribol. Lett. 45, 185 (2012).

[22] C. Creton and M. Ciccotti, Rep. Prog. Phys. 79, 046601 (2016).

[23] B. N. J. Persson, O. Albohr, G. Heinrich, and H. Ueba, J. Phys. Condens. Matter 17, R1071 (2005).

[24] K. Kendall, Molecular Adhesion and its Applications: The Sticky Universe (Kluwer Academic, New York, 2001).

[25] B. N. J. Persson and M. Scaraggi, J. Chem. Phys. 141, 124701 (2014).

[26] K. L. Johnson and K. Kendall, and A. D. Roberts, Proc. R. Soc. A 324, 301 (1971).

[27] M. Deruelle, H. Hervet, G. Jandeau, and L. Leger, J. Adhes. Sci. Technol. 12, 225 (1998).

[28] B. N. J. Persson and E. A. Brener, Phys. Rev. E 71, 036123 (2005).

[29] B. N. J. Persson, Eur. Phys. J. E 8, 385 (2002).

[30] J. A. Greenwood and K. L. Johnson, Philos. Mag. 43, 697 (1981).

[31] D. Maugis and M. Barquins, J. Phys. D: Appl. Phys. 11, 1989 (1978).

[32] The instrumental error bars in the data in Fig. 4(b) is mainly due to the finite rate at which we collect mass data, namely, 1 data point per second ( $\tau=1 \mathrm{~s}$ ). This imply that if the interaction force $F(t)$ is negative during some time interval $\Delta t$ there will be a maximal uncertainty in the pull-off force by a factor $\tau / \Delta t$. For the highest preload we have $\Delta t \approx 40 \mathrm{~s}$ so the maximum error is about $3 \%$. For the smallest preload $\Delta t \approx 8 \mathrm{~s}$ giving the maximum error $\sim 12 \%$.

[33] B. Lorenz, Y. R. Oh, S. K. Nam, S. H. Jeon, and B. N. J. Persson, J. Chem. Phys. 142, 194701 (2015).

[34] E. Barthel and C. Fretigny, J. Phys. D 42, 195302 (2009).

[35] T. L. Sun, F. Luo, W. Hong, K. Cui, Y. Huang, H. J. Zhang, D. R. King, T. Kurokawa, T. Nakajima, and J. P. Gong, Macromolecules 50, 2923 (2017).

[36] J. A. Greenwood, J. Phys. D 37, 2557 (2004).

[37] J. A. Greenwood, J. Phys. D 40, 1769 (2007).

[38] J. A. Greenwood, K. L. Johnson, S.-H. Choi, and M. K. Chaudhury, J. Phys. D 42, 035301 (2009).

[39] www.accudynetest.com/polymer_surface_data/butyl_rubber .pdf https://www.accudynetest.com/polytable_01.htm.

[40] The crack tip velocity at the onset of the pull-off instability (where the pull-off force $F=-F_{\mathrm{c}}$ ) depends on the pull-off force as $v_{r} \propto F_{\mathrm{c}}^{-1 / 3}$ [see Eq. (9) in Ref. [20]]. For bromobutyl in the relevant velocity region (around $100 \mu \mathrm{m} / \mathrm{s}$ ) $w(v) \sim v^{\beta}$, where $\beta \approx 0.2$. Hence, we expect the work of adhesion to depend on the preload as $w(v) \propto F_{c}^{-\beta / 3}=F_{c}^{-1 / 15}$. This weak 
dependency of $w(v)$ on $v$ can be neglected and is not included in Fig. 4.

[41] G. J. Lake and A. G. Thomas, Proc. R. Soc. A 300, 108 (1967).

[42] A. Schallamach, Wear 6, 375 (1963).

[43] S. Zilberman and B. N. J. Persson, J. Chem. Phys. 118, 6473 (2003).

[44] Q. Li and K.-S Kim, Acta Mech. Solida Sin. 22, 377 (2009).
[45] N. Mulakaluri and B. N. J. Persson, Europhys. Lett. 96, 66003 (2011).

[46] G. Carbone, E. Pierro, and G. Recchia, Phys. Rev. E 92, 062404 (2015).

[47] M. Müser, Tribol. Int. 100, 41 (2016).

[48] A. Tiwari, L. Dorogin, A. I. Bennett, K. D. Schulze, W. G. Sawyer, M. Tahir, G. Heinrich, and B. N. J. Persson, Soft Matter 13, 3602 (2017). 